\title{
Self-organization of domain growth in the Ising model with impurities
}

\section{Andersen, Jørgen Vitting; Mouritsen, Ole G.}

\section{Published in:}

Physical Review A

Link to article, DOI:

10.1103/PhysRevA.45.R5331

Publication date:

1992

Document Version

Publisher's PDF, also known as Version of record

Link back to DTU Orbit

Citation (APA):

Andersen, J. V., \& Mouritsen, O. G. (1992). Self-organization of domain growth in the Ising model with impurities. Physical Review A, 45(8), R5331-R5334. https://doi.org/10.1103/PhysRevA.45.R5331

\section{General rights}

Copyright and moral rights for the publications made accessible in the public portal are retained by the authors and/or other copyright owners and it is a condition of accessing publications that users recognise and abide by the legal requirements associated with these rights.

- Users may download and print one copy of any publication from the public portal for the purpose of private study or research.

- You may not further distribute the material or use it for any profit-making activity or commercial gain

- You may freely distribute the URL identifying the publication in the public portal

If you believe that this document breaches copyright please contact us providing details, and we will remove access to the work immediately and investigate your claim. 


\title{
Self-organization of domain growth in the Ising model with impurities
}

\author{
Jørgen Vitting Andersen* and Ole G. Mouritsen \\ Department of Physical Chemistry, The Technical University of Denmark, Building 206, DK-2800 Lyngby, Denmark
}

(Received 20 December 1991)

\begin{abstract}
We have studied avalanchelike rearrangements of domain patterns in the two-dimensional Ising model with static impurities, which is quenched to low temperatures. When breaking the up-down symmetry of the spins by a small applied field, the mere fluctuation of a single spin eventually results in a cascade of spin flips at the domain boundaries. We have analyzed the lifetime and size distribution functions for the avalanches and related the results to the general phenomena of selforganized criticality and to experiments on cellular magnetic domain patterns in magnetic garnet films. Our results suggest that the self-organized state in this system appears to be subcritical, in agreement with theoretical predictions.
\end{abstract}

PACS number(s): $5.40 .+\mathrm{j} ; 75.60 . \mathrm{Ch} ; 75.10 . \mathrm{Hk}$

Self-organizing dynamics of two-dimensional magnetic domain patterns has recently been studied in an experimental setup with magnetic garnet films [1], as well as in a theoretical study of a random-neighbor model [2]. In the experiment it was observed that over a significant interval of applied magnetic field, the magnetic domain pattern self-organizes into a state of barely stable configurations. In this state, small field increments trigger avalanches of cell destruction that span more than two orders of magnitude in size and lifetime. The experimental distributions of avalanche sizes and lifetimes appeared to follow power laws, and it was suggested that the system might have arranged itself into a selforganized critical state [3]. Later theoretical work [2] has described the dynamical properties of these domain patterns within a mean-field-type random-neighbor model, where it is found that the domain pattern self-organizes in a subcritical state. The avalanches of topological rearrangements and cell destruction in the random-neighbor model were found to obey power laws with mean-field exponents and exponential damping factors. Furthermore, the authors of Ref. [2] reanalyzed the experimental data of Ref. [1] and found the data to be consistent with the theoretical distributions, thus suggesting that the experimental system is also in a subcritical state.

In this paper we go beyond the mean-field description and allow fully for thermal fluctuations by presenting a computer-simulation calculation of the dynamical selforganization of domain patterns in a two-dimensional ferromagnetic Ising spin model which thermally has been quenched into its unstable regime. Being far from thermodynamic equilibrium in a symmetry-broken state, the system undergoes an ordering process which leads to a domain pattern which evolves in time. The pattern is composed of two domain types corresponding to the ferromagnetically ordered spin-up and spin-down states. Since the domain growth in the Ising model with nonconserved order parameter is fast and described by the Lifshitz growth law $R(t) \sim t^{1 / 2}$ where $R(t)$ is the linear dimension of the growing domain [4], we have studied the model in the presence of a static dispersion of nonmag- netic impurities (vacancies) which is known to slow down the domain growth and lead to a logarithmic growth law [5] $R(t) \sim \ln t$. This much slower growth permits a study of the self-organization of the domain pattern without a strong interference with the overall domain-growth process. In fact, on the time scale which characterizes the avalance effects at the domain boundaries, cf. the insert of Fig. 1, the overall domain-growth process may be considered as almost quasistatic. By driving the system very slowly using a uniform magnetic field and by only studying the dynamics within a time period that is negligible compared to the time required for the system to reach equilibrium, we anticipate a dynamical situation which is analogous to the dynamics in conventional self-organized critical systems, which are slowly driven steady-state systems [3]. It should be emphasized that the dynamics of domain formation in the impure Ising models is not a realistic model of the magnetic domain formation in magnetic garnet films. However, as pointed out above, we anticipate and show below that our simple model shares some very general dynamical features with the experimental system related to self-organized dynamical systems.

The two-dimensional Ising Hamiltonian is written as

$$
H=-J \sum_{\langle i, j\rangle} \sigma_{i} \sigma_{j},
$$

where the spin $\sigma_{i}$, in addition to the usual values -1 and 1 , also can take on the value 0 . The summation runs over nearest-neighbor sites on a square lattice. The $\sigma=0$ case is interpreted as a nonmagnetic impurity (vacancy). The interaction constant is ferromagnetic, $J>0$. The impurities are static and randomly dispersed in the lattice in a concentration of $c$. Using standard Monte Carlo techniques involving Glauber dynamics, the time-evolution of the model is studied after deep thermal quenches from the disordered phase into the ferromagnetically ordered phase. The time is measured in units of Monte Carlo steps per site (MCS/S).

The domain growth in the two-dimensional Ising model with quenched impurities has previously been studied in 
detail by Srolovitz and Hassold [6], who, using Monte Carlo computer simulation, found a crossover from an initial algebraic domain grow th to a late-time logarithmic growth when the model is quenched to very low temperatures, $k_{B} T / J=0.04 \ll k_{B} T_{\mathrm{c}} / J$, where $T_{\mathrm{c}}$ is the phase transition temperature. The time at which the crossover occurs depends on the concentration of impurities, with an earlier crossover to logarithmic growth for increasing impurity concentration.

In the present work we allow the system to evolve during sufficient time in order to assure that the logarithmic growth regime has been attained. The system is quenched from an initially disordered state $(T \sim \infty)$ to a very low temperature. Different lattice sizes are studied. As an example, for a lattice with $L \times L=250 \times 250$ sites and an impurity concentration of $c=3 \%$, around $5 \times 10^{3}$ MCS/S are needed to reach the logarithmic regime. Having assured that the system is evolving logarithmically slowly, we then apply a small uniform magnetic field in order to break the up-down spin symmetry. When the field is applied, the growth is faster than logarithmic but still slow and described by an algebraic growth law with a very small exponent. To avoid the transient increased activity of spin flips which are either barely activated or not activated at all, we let the system evolve further, e.g., $500 \mathrm{MCS} / \mathrm{S}$, before we start sampling.

The definition of an avalanche and the way it is determined is as follows: every time a spin flip is performed, we record in an avalanche array its position and the time when it is flipped. After every Monte Carlo sweep (1 MCS/S) we search the avalanche array for active and extinct avalanches. A group of spins in the avalanche array which are connected via nearest-neighbor bonds, and which contain at least one spin whose flip time does not differ more than $\delta t$ from the actual Monte Carlo time, is considered as being an active avalanche. Spins in the avalanche array which are not connected via nearestneighbor bonds to any other spin whose flip time lies in the interval $[t-\delta t, t]$ are considered as being extinct and are removed from the avalanche array. The number of spins $s$ in an extinct avalanche is recorded as an avalanche of size $s$, and the largest difference between the flip time of two spins in an extinct avalanche is recorded as the lifetime $t$ of the avalanche. A small value of $\delta t$ is required to ensure that all the nearest neighbors of a flipped spin are visited before the recording of the flipped spin eventually is deleted from the avalanche array. This is due to the random way in which the sites in the lattice are visited via the Metropolis method. Using a too large value of $\delta t$ increases the possibility that a new avalanche, which is initiated close to a newly extinct avalanche, will be counted together as a single avalanche. Due to the slow growth kinetics in the system, we have found that with a suitable chosen value of $\delta t$, e.g., $\delta t \approx 5 \mathrm{MCS} / \mathrm{S}$, we do not encounter problems of this kind. In order to obtain sufficient statistics within the short time period used, an average over several samples has to be performed. Usually we have averaged over 20-100 samples.

Since we are interested in the dynamics of a slowly driven system that can be thought of as quasistatic, the sampling takes place within a very short time pe- riod during which we can assume that the probability for the occurrence of an avalanche of a given size and a given lifetime is independent of time. Since the lifetime and the size of an avalanche is dependent on the extent of the perimeter of the formed domains, the time period has been chosen so that the change in the domaindistribution function during this time period can be neglected. Since the quench temperature is low, avalanches are initiated and propagate only from the perimeter of the domains and not from the interior of the domains. It is possible to average over longer time periods provided that the avalanche-size distribution function is corrected for the background domain growth. This can be done by identifying the growth law in effect and then scale the avalanche-size distribution function accordingly. The results obtained using this procedure are similar to those reported below obtained from short-time averaging.

In Fig. 1 is shown on a log-log plot the distribution of avalanche sizes $D(s)$ versus size $s$ of the avalanches for three different lattice sizes. The corresponding lifetime distribution, $D(t)$, of avalanches is shown on a log-log plot in Fig. 2. The quench temperature is $k_{B} T / J=0.07$ and the impurity concentration is $c=0.1$. The three different system sizes have been run for different times before the sampling starts since the crossover time to logarithmic growth is size dependent [6]. The applied field $H / J=0.7$ is the same for all system sizes and the field is applied after the system has reached the logarithmic growth regime. As seen in Figs. 1 and 2 the size dependence of the data is very weak. The statistics of the larger systems are of course better since it is possible to follow the larger systems to later times which in turn lead to better statistics for the large avalanches. It is important to note that it has been found that $D(s)$ and $D(t)$ are invariant to the time at which the sampling begins and also invariant to the size of the lattice. However, a given

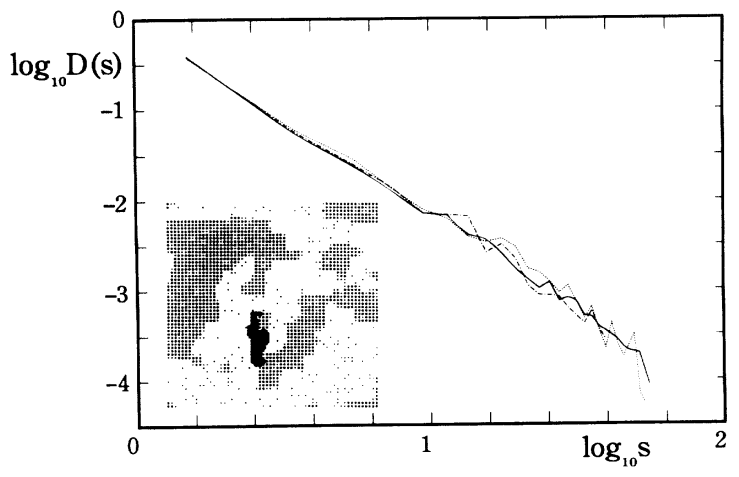

FIG. 1. Log-log plots of the distribution of avalanche sizes $D(s)$ vs size $s$ of an avalanche. The temperature is $k_{B} T / J=$ 0.07 , the impurity concentration is $c=0.1$, and the applied field is $H / J=0.7$. Results are shown for three different lattice sizes, $L \times L$. Dashed line: $50 \times 50$; dotted line: $100 \times 100$; solid line: $200 \times 200$. The insert shows a snapshot of a typical avalanche of a size around 50 spins. White areas correspond to down-spins, grey areas correspond to up-spins, and the black area denotes the spins involved in the avalanche. Small dots denote the static impurities. 


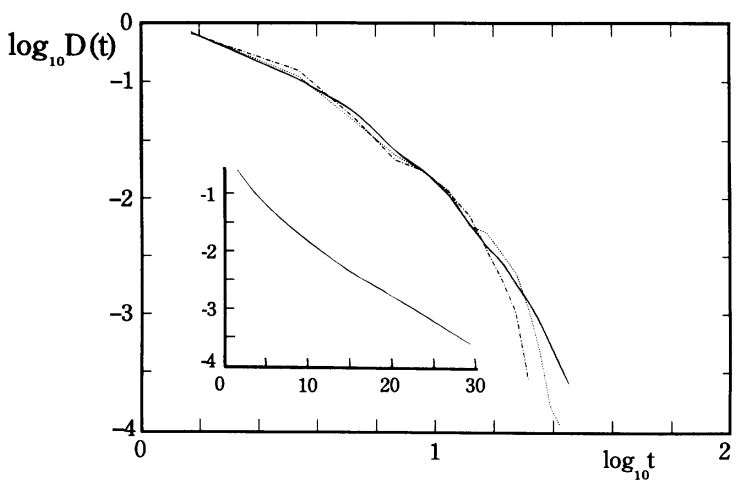

FIG. 2. Log-log plots of the distribution of avalanche lifetimes $D(t)$ vs lifetime $t$ for avalanches corresponding to Fig. 1 . The inset shows the same data in a semilogarithmic plot which demonstrates the long-time exponential behavior of the lifetime distribution.

lattice size at a given time provides better statistics to a certain part of the distributions.

Figures 1 and 2 show that the size-distribution function $D(s)$ is reasonably well described by an effective power law over one and a half decades, whereas the lifetime distribution $D(t)$ is better described by a power law times an exponential damping function. Adopting the general expression of an algebraic function times an exponential for both distribution functions, we can fit the data in Fig. 1 as

$D(s) \propto s^{-2.1} \exp \left(-s / s_{0}\right), \quad D(t) \propto t^{-0.6} \exp \left(-t / t_{0}\right)$

with $t_{0} \approx 6$ and $s_{0} \sim \infty$. Hence, it appears that the dynamics of the domain formation in this model has a characteristic time scale whereas no characteristic length scale can be identified. The long-time exponential behavior of the lifetime distribution function is demonstrated in the insert of Fig. 2. Consequently, the system is in a subcritical state with effective power-law behavior in the avalanche-size distribution function. We have studied the model at other temperatures, other impurity concentrations $(c \simeq 3-10 \%)$, and for other values of the applied magnetic field and have found that, except at high temperatures and high magnetic fields, the behavior is qualitatively the same, Eq. (2), but with different values of $t_{0}$. Hence the subcritical self-organization of the system is robust. It should be noted that the weak, but systematic size dependence seen in Fig. 2 might indicate that for extremely large systems the exponential damping may go away. A similar, extremely weak size dependence has been found in the cellular automaton, the Game of Life, which is a simple algorithm for the survival in space and time of a species [7].

We have analyzed the power spectrum $S(f)$ of the time series of the total avalanche activity $N(t)=\sum s(t)$ defined as the superposition of the independent avalanches currently in progress:

$$
S(f)=\left|\int N(t) \mathrm{e}^{-2 \pi i f t} \mathrm{~d} t\right|^{2} .
$$

The simulation results for three different system sizes are



FIG. 3. Power spectrum $S(f)$ of time series corresponding to the data of Figs. 1 and 2. The solid line denotes the function $S(f) \sim 1 / f^{09}$. Results are shown for three different lattice sizes, $L \times L$. Dotted line: $50 \times 50$; dashed line: $100 \times 100$; solid line: $200 \times 200$.

shown in Fig. 3. The spectra for the different systems are almost identical apart from a trivial size-dependent scaling factor. The overall behavior of the power spectrum for small frequencies shows a crossover to white-noise behavior and for higher frequencies an effective power-law scaling. The scaling regime extends over approximately one decade, and the power law is given by $S(f) \sim 1 / f^{\beta}$, with $\beta \simeq 0.9$. The finding of effective $1 / f$ noise behavior in the power spectrum in a domain-growth problem is consistent with the finding by Roland and Grant [8] of a similar behavior with $\beta \simeq 0.9 \pm 0.05$ for the kinetics of domain growth in the standard two-dimensional Ising model. These authors analyzed the fluctuations in the ordering kinetics by Monte Carlo simulation in terms of a probability measure for the interfacial domain-boundary dynamics and showed that the fluctuations display nonself-averaging behavior which gives rise to multiscaling and $1 / f$ noise. These results are of a rather general nature and closely related to recent findings of $1 / f$-noiselike behavior in nonequilibrium and steady-state driven diffusive systems [9-11].

In conclusion, we have shown that the avalanchelike rearrangements of domain patterns in the impure Ising model driven quasistatically by a weak uniform magnetic field have the signature of a self-organized state which is subcritical. The avalanche-size distribution function displays, however, an effective power-law behavior and the power spectrum is $1 / f$-noise-like. Our results are in accordance with similar findings from a random-neighbor mean-field model study [2] and an experiment [1] on the dynamics of domain patterns in magnetic garnet films. It is interesting to note that a recent experiment [12] on a related effect, the Barkhausen effect, in an amorphous alloy showed self-organized critical behavior with a power spectrum characterized by exponent values in the range $\beta \sim 1.1-1.5$, depending on the treatment of the alloy.

This work was supported by the Danish Natural Science Research Council under Grant Nos. 11-7785 and 11-9319-1, and by the Danish Technical Research Council under Grant No. 16-4296.K. J.V.A. wishes to acknowledge support from the Maxwell Foundation. 
* Present address: Laboratoire de Thermodynamique Physico-Chimie Metallurgiques, Institut National Polytechnique de Grenoble, ENSEEG Domaine Universitaire, Boîte Postale 75, F-38402 Saint-Martin-d'Heres CEDEX, France.

[1] K. L. Babcock and R. M. Westervelt, Phys. Rev. Lett. 64, 2168 (1990).

[2] P. Bak and H. Flyvbjerg, Phys. Rev. A 45, 2192 (1992).

[3] P. Bak, C. Tang, and K. Wiesenfeld, Phys. Rev. Lett. 59, 381 (1987); Phys. Rev. A 38, 364 (1988).

[4] O. G. Mouritsen, in Kinetics of Ordering and Growth on Surfaces, edited by M. G. Lagally (Plenum, New York, 1990), p.1.
[5] D. A. Huse and C. L. Henley, Phys. Rev. Lett. 54, 2708 (1985).

[6] D. J. Srolovitz and G. N. Hassold, Phys. Rev. B 35, 6902 (1987).

[7] P. Bak (private communication).

[8] C. Roland and M. Grant, Phys. Rev. Lett. 63, 551 (1989).

[9] H. J. Jensen, Phys. Rev. Lett. 64, 3103 (1990).

[10] J. V. Andersen, H. J. Jensen, and O. G. Mouritsen, Phys. Rev. B 44, 439 (1991).

[11] K.-t. Leung, Phys. Rev. B 44, 5340 (1991).

[12] P. J. Cote and L. V. Meisel, Phys. Rev. Lett. 67, 1334 (1991). 


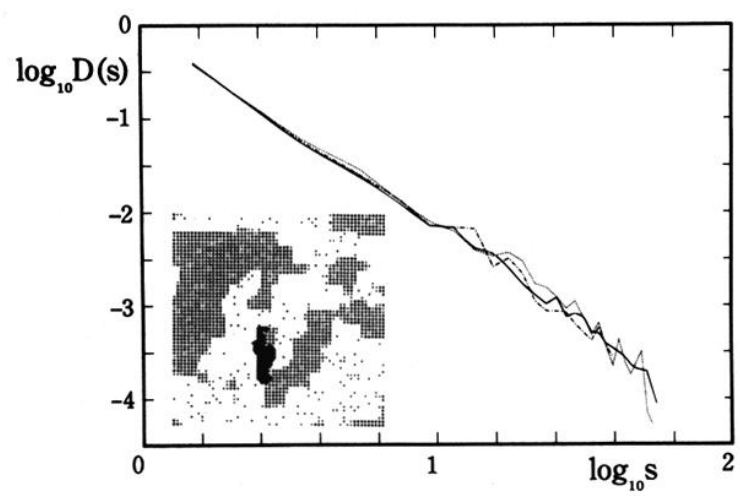

FIG. 1. Log-log plots of the distribution of avalanche sizes $D(s)$ vs size $s$ of an avalanche. The temperature is $k_{B} T / J=$ 0.07 , the impurity concentration is $c=0.1$, and the applied field is $H / J=0.7$. Results are shown for three different lattice sizes, $L \times L$. Dashed line: $50 \times 50$; dotted line: $100 \times 100$; solid line: $200 \times 200$. The insert shows a snapshot of a typical avalanche of a size around 50 spins. White areas correspond to down-spins, grey areas correspond to up-spins, and the black area denotes the spins involved in the avalanche. Small dots denote the static impurities. 